\title{
Use of hem-o-lok clips for laparoscopic appendectomy in children: retrospective analysis and comparison to ligature loop and endoscopic surgical stapler
}

\author{
Ennio J. Fuentes ${ }^{1}$, Eduardo A. Pérez ${ }^{2}$, Ricardo Díez ${ }^{1}$, Pablo Aguado ${ }^{1}$, Cecilia Moreno ${ }^{1}$, Henar Souto ${ }^{3}$, \\ Jose L. Alonso ${ }^{3}$ \\ ${ }^{1}$ Department of Pediatric Surgery, University Hospital, Fundación Jiménez Díaz, Madrid, Spain; ${ }^{2}$ Division of Pediatric Surgery, University of Miami \\ Miller School of Medicine, Miami, Florida, USA; ${ }^{3}$ Department of Pediatric Surgery, University Children's Hospital of the Niño Jesús, Madrid, Spain \\ Contributions: (I) Conception and design: EJ Fuentes; (II) Administrative support: H Souto, JL Alonso; (III) Provision of study materials or patients: \\ EJ Fuentes; (IV) Collection and assembly of data: EJ Fuentes, JL Alonso; (V) Data analysis and interpretation: JL Alonso; (VI) Manuscript writing: \\ All authors; (VII) Final approval of manuscript: All authors. \\ Correspondence to: Prof. Dr. Jose L. Alonso, MD. Jefe Departamento de Cirugía Pediátrica, Hospital Infantil Universitario Niño Jesús, Avenida \\ Menendez Pelayo, 65, 28009, Madrid, Spain. Email: jlorenzo.alonso@salud.madrid.org.
}

Background: There are multiple techniques used for laparoscopic appendectomy (LA): ligature loop (LL), surgical stapler (SS) (Endopath Ets-Flex-Endoscopic Articulating Linear Cutter 33 mm Standard Ref Atb 35. Ethicon, Somerville, New Jersey, US), and hem-o-lok clips (HOL) (Weck Closure System. Triangle Park, NC, USA). The application of the LL usually demands dexterity and training, whilst using HOL may be more advantageous due to its simplicity in terms of application and its low cost in contrast with the SS. The objective of this study is to determine safety and efficacy of the different devices that can be used in the surgical procedure.

Methods: From June 2016 to December 2019, 253 consecutive children aged to 1 to 18 years were retrospectively reviewed. They were divided into three groups depending on the device used to secure the appendix: (I) in the first group, the base of the appendix was secured by double LL, (II) in the second group the base of the appendix was secured with SS, and (III) in the third group the base of the appendix was secured with two non-absorbable HOL. The data collected includes age, gender, operative time, device used to ligate the base of the appendix, previous tests (blood analysis, imaging), antibiotic prophylaxis administered, length of hospital stay, intraoperative and postoperative complications, shoulder pain and histological study of the specimen.

Results: There were 253 patients that underwent laparoscopic appendectomy during the study time, with a mean age of $10.3 \pm 4.1$ in the LL group, $9.4 \pm 2.7$ in the SS group and $10.4 \pm 3.3$ in the HOL group, $\mathrm{P}=0.165$. Distribution by gender was $77.8 \%$ for males in the LL group, $65.2 \%$ in the SS group and $61.3 \%$ in the HOL group, $\mathrm{P}=0.559$. The mean surgical time with $\mathrm{IQR}$ in brackets was 60.0 (10.0) minutes ( $\mathrm{min}$ ), in the first group in which the base of the appendix was secured with LL, in the second group in which the base of the appendix was secured with SS 60.0 (15.0) min and finally in the third in which the base of the appendix was secured with HOL 40.0 (30.0) $\mathrm{min}, \mathrm{P}<0.001$. HOL clips have a significantly lower cost than their analogues. Specifically, 5 HOL clips have a cost of EUR 26.75, while three LL have a cost of EUR 53.70 and a single SS has a cost of EUR 276.58. Postoperative complications were found in $14.3 \%$ of the LL group, $9.8 \%$ in the SS group and $4.6 \%$ in the HOL group, $\mathrm{P}=0.137$. Efficacy and safety in controlling the base of appendix were the same in all groups.

Conclusions: The HOL are safe and reduce surgical costs during laparoscopic appendectomy in children.

Keywords: Appendectomy; appendicitis; children; laparoscopy; hem-o-lok clip (HOL)

Received: 05 May 2020; Accepted: 21 August 2020; Published: 25 July 2021.

doi: $10.21037 /$ tgh-20-213

View this article at: http://dx.doi.org/10.21037/tgh-20-213 


\section{Introduction}

Acute appendicitis is one of the most common causes of acute abdomen in childhood, requiring surgical treatment in the vast majority of cases (1). Traditionally its surgical treatment has consisted of an open appendectomy, however after the advent of laparoscopic techniques, especially in the last decades, laparoscopic appendectomy has become the standard surgical treatment in children and adolescents, especially in uncomplicated appendicitis. Its advantages over the open technique are well known, achieving a rapid recovery, less postoperative pain and greater patient comfort, although some cases experience pain in the right shoulder due to phrenic irritation (2).

Traditionally, the laparoscopic approach has involved three ports with intracorporeal dissection and ligation of the appendix and mesoappendix. In the last few years, an extracorporeal approach through an umbilical port or two ports (umbilical and suprapubic) has also been proposed to facilitate the externalization of the appendix. Single port techniques with an operative scope or the single site appendectomy have also been described by surgeons in order to perform this common procedure $(3,4)$.

In the intracorporeal approach, various devices have been used to ligate and secure the base of the appendix during this time. Three of these devices have been used in the treatment of the patients included in this study: ligature loop (LL) (PDS II Violet Monofilament Polidioxanone Suture $0(53 \mathrm{~cm})$ Ethicon Somerville, New Jersey, USA, surgical stapler (SS) (Endopath Ets-Flex-Endoscopic Articulating Linear Cutter $33 \mathrm{~mm}$ Standard Ref Atb 35. Ethicon, Somerville, New Jersey, USA) and hem-o-lok clips (HOL) (Weck Closure System. Triangle Park, NC, USA).

The purpose of this study is to compare different devices used to control the base of the appendix and their reliability. Specifically, the safety and efficacy of the HOL.

We present the following article in accordance with the STROBE reporting checklist (available at http://dx.doi. org/10.21037/tgh-20-213).

\section{Methods}

The study was conducted in accordance with the Declaration of Helsinki (as revised in 2013). The study was approved by the institutional ethics committee of the University Hospital, Fundación Jiménez Díaz (UHUFJD) (No. EO126-20_FJD) and individual consent for this retrospective analysis was waived.
From June 2016 to December 2019, 253 consecutive children aged to 1 to 18 years underwent a laparoscopic appendectomy. On all occasions the procedure was performed by the same group of 3 surgeons. Sixty three percent were male $(n=159)$. All the patients who underwent the aforementioned surgery presented with a clinical picture of acute appendicitis. Patients underwent laboratory work up and imaging. Preoperative antibiotics were administered to $88.6 \%$ of patients, using intravenous antibiotics at prophylactic doses according to the standard protocol of institution established for this purpose (amoxicillin/ clavulanate $100 \mathrm{mg} / \mathrm{kg} /$ day in uncomplicated appendicitis or amoxicillin/clavulanate $100 / \mathrm{mg} / \mathrm{kg} / \mathrm{day}+$ gentamicin $5 \mathrm{mg} / \mathrm{kg} /$ day in complicated appendicitis, which was defined by the presence of perforation of the appendix, local phlegmon or abscess or diffuse peritonitis).

The patients were divided into three groups, according to the device used to ligate the base of the appendix. This was done based on surgeon preference and not randomized. The first group received double LL, while the SS was used in the second, and the base was secured by a two large nonabsorbable clips (HOL) in the third group (Figure 1).

Patients were not included in the study if they had a laparoscopic appendectomy with another device such as metal clips. Likewise, they were not included if the procedure was performed by a surgeons that did not belong to the ones that performed the study.

The patient was placed in a supine position combined with the Trendelenburg position and left lateral position. The surgeon and assistant stood to the left side of the patient, and the monitor was on the patient's right side. The bladder was decompressed to avoid injury during insertion of suprapubic ports. Three laparoscopic trocars were placed: one $12 \mathrm{~mm}$ trocar at the umbilicus, one $5 \mathrm{~mm}$ trocar at the midline just cephalic to the pubic bone, and one $5 \mathrm{~mm}$ at the left iliac fossa (Figure 2). The umbilical port was used to remove the specimen.

The data collected includes information regarding age, gender, operative time, device used to ligate the base of the appendix, work up (blood analysis, imaging), antibiotic prophylaxis, length of hospital stay, intraoperative and postoperative complications, and histological interpretation of the appendectomy specimen (Tables 1,2).

Quantitative variables were summarized by mean and standard deviation, or by median and interquartile range, and qualitative variables were summarized by absolute and relative frequencies. Variables described by mean and standard deviation were compared by one-way analysis of 


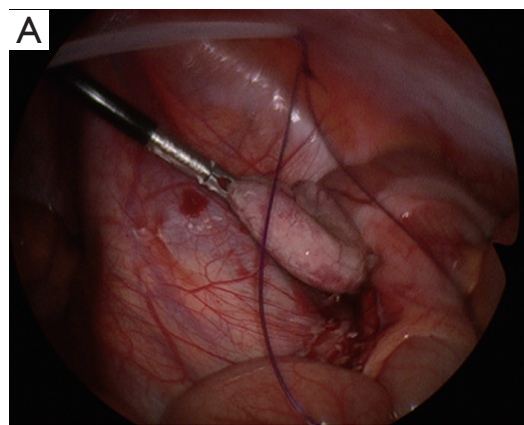

Hem-o-lok, endoloop and surgical stapler
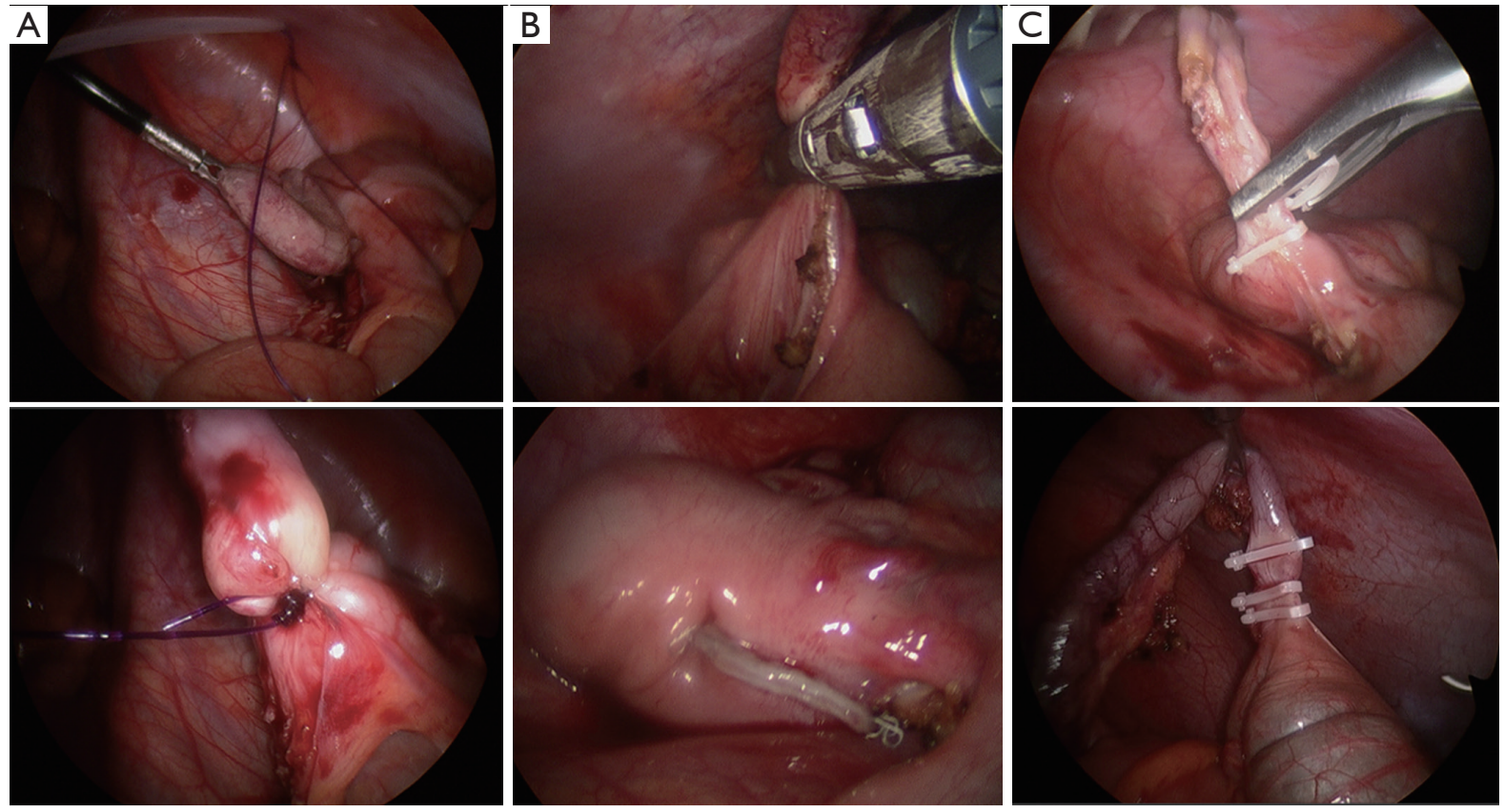

Figure 1 Laparoscopic appendiceal base devices used. (A) Loop ligatures (LL); (B) surgical stapler (SS); (C) hem-o-lok clips (HOL).

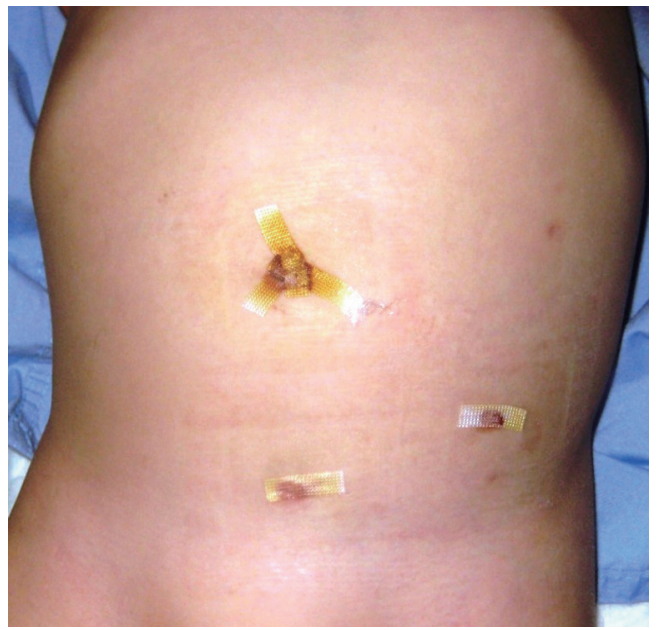

Figure 2 Laparoscopic ports in acute appendicitis (umbilical, suprapubic and left iliac fossa).

variance, while those described by median and interquartile range were compared by Kruskal-Wallis test. For comparisons of qualitative variables the Chi-square test or the Fisher's exact test, were performed. In all comparisons the significance level was set at 0.05 . Statistical analysis was performed using $\mathrm{R}$ version 3.6.3 (R Foundation for
Statistical Computing, Vienna, Austria) (Tables 1,2).

\section{Results}

Overall, 253 consecutive laparoscopic appendectomies were performed using the surgical technique described, of which two required change of surgical technique: (I) one case due to an unknown intestinal malrotation in an obese patient, and (II) another for complexity of dissection due to the presence of a subhepatic localized peritonitis (Table 2).

The LL group consisted of 9 patients (3.5\%), while the SS group had 46 (18.1\%), and the HOL group had 199 patients $(78.3 \%)$. Mean patient age was 9.2 years (range, 1 to 18 years) and the ratio males to females was 159:95 respectively. The mean age was $10.3 \pm 4.1$ in the LL group, $9.4 \pm 2.7$ in the SS group and $10.4 \pm 3.3$ in the HOL group, $\mathrm{P}=0.165$. The gender distribution of male patients was $77.8 \%$ in the LL group, $65.2 \%$ in the SS group and $61.3 \%$ in the HOL group, $\mathrm{P}=0.559$ (Table 1).

Patients underwent laboratory work up which revealed leukocytosis, elevated CRP or other inflammatory markers (such as procalcitonin) in $74.7 \%$ of cases, $55.6 \%$ in the LL group, $82.2 \%$ in the SS group and $85.3 \%$ in the HOL group, $\mathrm{P}=0.199$. Imaging studies revealed alterations in 
Table 1 Demographic and preoperative test variables

\begin{tabular}{|c|c|c|c|c|}
\hline Variable & Loop ligature (1A) & Surgical stapler (1B) & Hem-o-lok (1C) & $P$ value \\
\hline Gender (male) & $7(77.8 \%)$ & $30(65.2 \%)$ & $122(61.3 \%)$ & 0.559 \\
\hline Blood analysis (abnormal) & 4 (44.4\%) & $37(82.2 \%)$ & $146(73.4 \%)$ & 0.199 \\
\hline Imaging tests & $6(75.0 \%)$ & $43(95.6 \%)$ & 194 (98.0\%) & 0.018 \\
\hline
\end{tabular}

Table 2 Surgical variables

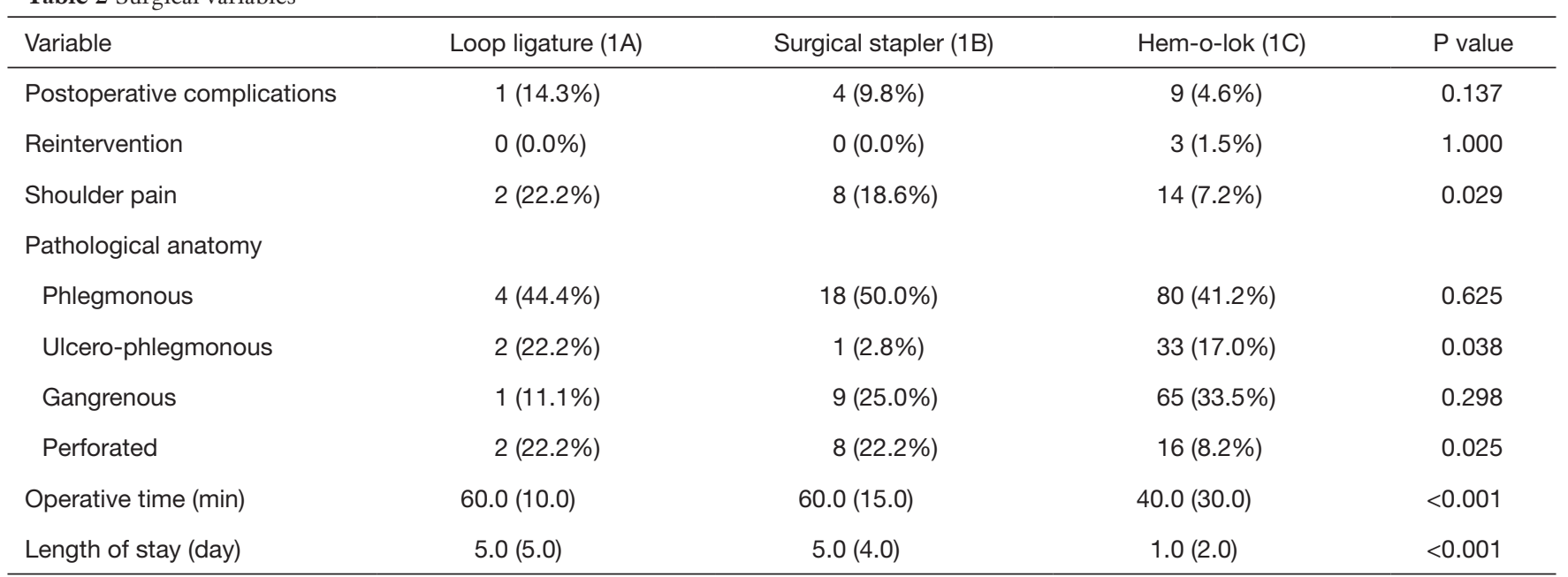

$85.7 \%$ of the patients, $86.7 \%$ in the LL group, $88.4 \%$ in the SS group and $85.3 \%$ in the HOL group, $\mathrm{P}=0.018$ (Table 1).

The mean surgical time was $60.0(10.0) \mathrm{min}$ in the first group in which the base of the appendix was secured with LL, 60.0 (15.0) $\mathrm{min}$ in the second group in which the base of the appendix was secured with SS and finally 40.0 (30.0) min in the third in which the base of the appendix was secured with HOL, $\mathrm{P}<0.001$. Surgical time is described by median and interquartile range (between brackets) because the data are not normally distributed (Table 2).

The price of the devices used in each case to control the base of the appendix were: five HOL amount to EUR 26.75, compared with the three LL which amount to EUR 53.70 and surgical staples which amount to EUR 276.58.

There were no intraoperative complications. Postoperative complications were intraabdominal abscesses in most cases and presented in $14.3 \%(\mathrm{n}=1)$ of patients in the LL group, $9.8 \%(n=4)$ in the SS group and $4.6 \%(n=9)$ in the HOL group, $\mathrm{P}=0.137$. Of the 9 patients with intraabdominal abscess in the HOL group 3 patients presented with intestinal obstruction (Table 2). All cases were initially treated with broad-spectrum antibiotics, according to the standard protocol established for this purpose (amoxicillin/ clavulanate $100 / \mathrm{mg} / \mathrm{kg} / \mathrm{day}+$ gentamicin $5 \mathrm{mg} / \mathrm{kg} / \mathrm{day}$ ). If not responding appropriately, they required conversion to a different antibiotic regimen (meropenem $60 \mathrm{mg} / \mathrm{kg} /$ day). Moreover, 3 patients required re-intervention in the HOL group due to (I) obstructive adhesions in one case, (II) intra-abdominal abscess refractory to antibiotic treatment in another case and (III) drainage of large wall abscess connected to an intraabdominal abscess in another case (Table 2).

Right shoulder pain was referred by $9.6 \%$ of patients postoperatively. Right shoulder pain was reported by $22.2 \%$ of patients in the LL group, $18.6 \%$ in the SS group and $7.2 \%$ in the HOL group, $\mathrm{P}=0.029$ (Table 2).

The mean length hospital stay was 2 days, with a range between 1 and 16 days. Average length was $5.0(5.0)$ in the LL group, 5.0 (4.0) in the SS group and $1.0(2.0)$ in the HOL group, $\mathrm{P}<0.001$ (Table 2).

The histopathological results of the appendectomy specimens analyzed were normal $(0.8 \%)$, phlegmonous 
(42\%), $\mathrm{P}=0.625$, phlegmonous-ulcer (14.8\%), $\mathrm{P}=0.038$, gangrenous $(31.7 \%), \mathrm{P}=0.298$ and perforated $(10.7 \%)$, $\mathrm{P}=0.025$ (Table 2).

\section{Discussion}

Appendectomy is one of the most common procedures performed by pediatric surgeons worldwide. In this study we looked at different methods of controlling the base of the appendix during laparoscopic appendectomy (Figure 1)

Our results are similar to other published literature on laparoscopic appendectomy in childhood with respect to the following variables: age, gender, preoperative tests, antibiotic prophylaxis, postoperative complications and histopathological analysis (Tables 1,2) (1,3-5).

The use of HOL, a non-absorbable polymer structure for ligation of vessels, ureters and bile ducts, has been widely documented. Many studies have been conducted in order to evaluate the efficiency of polymer clips in minimally invasive surgery. Some studies have shown that it is possible to apply a double HOL to secure the base of the appendix during a laparoscopic appendectomy (5-8), and many others have compared its effectiveness with LL and/or a stapler device in adults $(9,10)$.

It is important to note, that the application of LL usually requires certain dexterity and training, meanwhile the application of HOL is available for every surgeon without the need for prior use. Likewise, every HOL is applied at a $90^{\circ}$ angle to the base of the appendix, which has proven to be of great importance and usefulness in the surgery described (8). The SS, as with LL, requires some training prior to its use as well as sufficient space in the abdominal cavity which is not always possible in children.

In contrast to metallic clips, the axial and transverse force required to dislodge these polymer clips, has been proven to be higher (11-13).

The clip is a nonabsorbable inert polymer, nonconductive and radiolucent, and its tip is designed to retain the clip in applier jaws, with an integrated teeth interface to prevent slippage (Figure 1).

Their use shows a slight downward trend in operative time, although this is not statistically proven in view of the study's sample size. The mean operative time of laparoscopic appendectomy was less than in the HOL group, although it must be taken into consideration that this was the largest group, and as a result the lower surgical time cannot be attributed exclusively to the device used (Table 1) $(1,3,4)$.

Length of stay was significantly lower in the HOL group $(\mathrm{P}<0.001)$, even though this was the group with larger number of complications. Regardless, we cannot conclude that the use of HOL shortens the length of stay due to the fact that this is the largest group and is bias by its large number of subjects. Even more, patients on the LL and SS group presented with higher rates of complicated appendicitis making the length of stay less related to device used. At most it indicates a trend.

Analysis of the price of the device used to control the base of the appendix has shown that the cost of a $5 \mathrm{HOL}$ case is half that of $3 \mathrm{LL}$ and one tenth of the cost of SS. This, together with its greater ease of use and reliability, leads us to recommend the use of HOL in laparoscopic appendicitis procedures in children.

We have not found any references relating to children showing the incidence of right shoulder pain after laparoscopic appendectomy. However, there are many references about this in adults, even after undergoing different surgical procedures. This is true even when using techniques to minimize its occurrence (2).

The incidence of right shoulder pain in this study has been $9.6 \%$, without the use of any preventive techniques (Table 2).

Our data shows that the use of Hem-o-lock clips is safe, effective and its use reduces surgical costs when compared with other more common techniques.

We understand that there are several limitations in the study. These include the retrospective nature of the design and selection bias as seen by the difference in number of patients in each group, favoring the HOL. However, it can be used to establish the rationale for a randomized controlled trial on a potentially safe, efficacy and costreducing surgical technique.

\section{Acknowledgments}

Our gratitude for Ignacio Mahíllo Fernández (Unidad de Bioestadística y Epidemiología, Hospital Universitario Fundación Jiménez Díaz) due to your contribution in the statistical analysis of this report; and our gratitude for Paula Alonso Barrera and Oliver Shaw due to your contribution in translating this report.

Funding: None.

\section{Footnote}

Provenance and Peer Review: This article was commissioned by the editorial office, Translational Gastroenterology and 
Hepatology for the series "Current Topics in Pediatric General Surgery". The article has undergone external peer review.

Reporting Checklist: The authors have completed the STROBE reporting checklist. Available at http://dx.doi. org/10.21037/tgh-20-213

Data Sharing Statement: Available at http://dx.doi. org/10.21037/tgh-20-213

Conflicts of Interest: All authors have completed the ICMJE uniform disclosure form (available at http://dx.doi. org/10.21037/tgh-20-213). The series "Current Topics in Pediatric General Surgery" was commissioned by the editorial office without any funding or sponsorship. Dr. EAP served as the unpaid Guest Editor of the series and serves as an unpaid editorial board member of Translational Gastroenterology and Hepatology from Jan 2019 to Dec 2020. The authors have no other conflicts of interest to declare.

Ethical Statement: The authors are accountable for all aspects of the work in ensuring that questions related to the accuracy or integrity of any part of the work are appropriately investigated and resolved. The study was conducted in accordance with the Declaration of Helsinki (as revised in 2013). The study was approved by the institutional ethics committee of the University Hospital, Fundación Jiménez Díaz (UHUFJD) (No. EO126-20_FJD) and individual consent for this retrospective analysis was waived.

Open Access Statement: This is an Open Access article distributed in accordance with the Creative Commons Attribution-NonCommercial-NoDerivs 4.0 International License (CC BY-NC-ND 4.0), which permits the noncommercial replication and distribution of the article with the strict proviso that no changes or edits are made and the original work is properly cited (including links to both the formal publication through the relevant DOI and the license). See: https://creativecommons.org/licenses/by-nc-nd/4.0/.

\section{References}

1. Rentea RM, Peter SDS, Snyder CL. Pediatric appendicitis: state of the art review. Pediatr Surg Int 2017;33:269-83.

2. Bhattacharjee HK, Jalaludeen A, Bansal V, et al. Impact of standard-pressure and low-pressure pneumoperitoneum on shoulder pain following laparoscopic cholecystectomy: a randomised controlled trial. Surg Endosc 2017;31:1287-95.

3. Bence CM, Wu R, Somers KK, et al. A tiered approach to optimize pediatric laparoscopic appendectomy outcomes. J Pediatr Surg 2019;54:2539-45.

4. El-Beheiry M, Davidson J, Jones S, et al. Outcomes of extracorporeal, transumbilical versus intracorporeal laparoscopic appendectomy for acute uncomplicated appendicitis in children and adolescents: A retrospective observational cohort study. J Pediatr Surg 2019;54:1059-62.

5. Delibegović $\mathrm{S}, \mathrm{Mehmedovic} \mathrm{Z}$. The influence of the different forms of appendix base closure on patient outcome in laparoscopic appendectomy: a randomized trial. Surg Endosc 2018;32:2295-9.

6. Sürek A, Bozkurt MA, Akbulut S, et al. Hem-o-lok Clip Is a Safe Alternative in the Laparoscopic Treatment of Perforated Appendicitis. Am Surg 2019;85:e245-e246.

7. Soll C, Wyss P, Gelpke H, et al. Appendiceal stump closure using polymeric clips reduces intra-abdominal abscesses. Langenbecks Arch Surg 2016;401:661-6.

8. Knight SR, Ibrahim A, Makaram N, et al. The use of polymeric clips in securing the appendiceal stump during laparoscopic appendicectomy: a systematic review. Eur J Trauma Emerg Surg 2019;45:665-70.

9. Yildız I, Koca S. Is There An Ideal Stump Closure Technique In Laparoscopic Appendectomy? Surg Technol Int 2016;28:117-20.

10. Bozkurt MA, Ünsal MG, Kapan S, et al. Two different methods for appendiceal stump closure: metal clip and Hem-o-lok clip. J Laparoendosc Adv Surg Tech A 2014;24:571-3.

11. Ponsky L, Cherullo E, Moinzadeh A, et al. The Hemo-lok clip is safe for laparoscopic nephrectomy: a multiinstitutional review. Urology 2008;71:593-6.

12. Klein RD, Jessup G, Ahari F, et al. Comparison of titanium and absorbable polymeric surgical clips for use in laparoscopic cholecystectomy. Surg Endosc 1994;8:753-8.

13. Deans GT, Wilson MS, Brough WA. The ability of laparoscopic clips to withstand high intraluminal pressure. Arch Surg 1995;130:439-41.

doi: $10.21037 /$ tgh-20-213

Cite this article as: Fuentes EJ, Pérez EA, Díez R, Aguado P, Moreno C, Souto H, Alonso JL. Use of hem-o-lok clips for laparoscopic appendectomy in children: retrospective analysis and comparison to ligature loop and endoscopic surgical stapler. Transl Gastroenterol Hepatol 2021;6:44. 